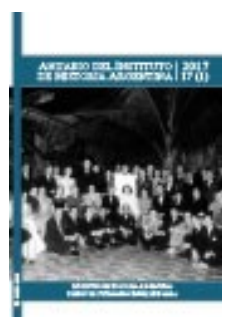

Anuario del Instituto de Historia Argentina, vol. 17, nº 1, e036, junio 2017.

ISSN 2314-257X

Universidad Nacional de La Plata.

Facultad de Humanidades y Ciencias de la Educación.

Centro de Historia Argentina y Americana

\title{
La Organización Internacional del Trabajo y la libertad sindical en América Latina: el caso de Venezuela en 1949
}

\author{
The International Labor Organization and Union freedom in Latin \\ America: The case of Venezuela in 1949
}

\section{Juan Carlos Yáñez Andrade *}

* Universidad de Valparaíso, Chile I juancarlos.yanez@uv.cl

\section{PALABRAS CLAVE}

OIT

Movimiento obrero

Libertad sindical

Venezuela

Asistencia técnica

KEYWORDS

ILO

Labor Movement

Union Freedom Conferences

Venezuela

Technical Assistance

\section{RESUMEN}

El presente artículo busca analizar las relaciones entre la Organización Internacional del Trabajo (OIT) y el movimiento sindical de América Latina. Si los intentos por fortalecer el movimiento de trabajadores eran de larga data, durante los años 1930 las relaciones entre el movimiento sindical latinoamericano y la OIT comenzaron a hacerse más regulares, especialmente en foros internacionales. Atención especial merece la misión de asistencia en materia sindical que llevó a cabo la OIT en Venezuela (1949), con el objeto de estudiar la situación de las organizaciones obreras luego de la instauración de la dictadura militar del general Carlos Delgado (1948). En términos de fuentes, se hará uso especialmente del informe redactado por la misión liderada por Jef Rens, subdirector general de la OIT, publicado en 1949, así como de la documentación presente en el archivo de la OIT en Ginebra.

\section{ABSTRACT}

This article analyzes the relationship between the International Labor Organization (ILO) and the trade union movement in Latin America. If attempts to strengthen the workers' movement were longstanding, during the 1930s relations between the Latin American trade union movement and the ILO began to become more regular, especially in international forums. Special attention deserves the assistance mission carried out by the ILO in Venezuela in 1949 to study the situation of workers' organizations after the establishment of the military dictatorship of General Carlos Delgado (1948). Document relevant is the report written by the mission led by Jef Rens, Assistant Director General of the ILO, and published in 1949. 


\section{Introducción}

El objetivo central de este artículo es analizar la relación entre la OIT y el movimiento sindical latinoamericano, en particular la importancia que comenzó a tener la defensa del principio de libertad sindical en el marco de gobiernos interesados en promover un sindicalismo proclive a sus intereses o que, directamente, implementaron medidas de persecución a su dirigencia.

La OIT fue creada en 1919 en el contexto del Tratado de Versalles y tuvo un destacado papel en la difusión de normas internacionales del trabajo $\underline{1}$. Si durante los años 1920 la OIT apostó por una política de ratificación de las convenciones internacionales del trabajo, a partir de fines de los años 1930 inició una política consistente en promover misiones de asistencia técnica, definidas como un "conjunto de métodos permitiendo aportar una ayuda en vista de la solución de un aspecto técnico cualquiera” (OIT, 1954, p. 3), lo que implicaba el desplazamiento de un funcionario o grupo de funcionarios expertos en materias técnicas con el fin de ofrecer una asistencia en el dominio de sus competencias. La lentitud en el proceso de ratificación por parte de los países latinoamericanos, junto a las demandas por implementar asesorías que orientaran los procesos de reforma social de acuerdo a estándares internacionales explican la confluencia de intereses de las autoridades nacionales y de los funcionarios de la OIT en torno a la asistencia técnica.

Para la OIT la promoción de un sindicalismo fuerte, con una relativa autonomía de los gobiernos, era importante en la consolidación de sus nexos con los trabajadores del continente y en la participación regular de las organizaciones obreras en las conferencias internacionales del trabajo. Según el modelo de representación tripartita instaurado por la Constitución de la OIT, las disposiciones internacionales que reglamentaban las condiciones de trabajo debían ser aprobadas de acuerdo a un sistema de doble discusión en el que los delegados de los gobiernos, organizaciones empresariales y sindicales consensuaban sus posiciones. De esta forma, la ausencia de los trabajadores latinoamericanos de las primeras conferencias internacionales fue un aspecto que preocupó al primer director de la OIT, Albert Thomas, que lo llevó a visitar el Cono Sur de América en 1925 (Yáñez, 2000; Ferreras, 2011).

Si bien América Latina había sido una región importante para el desarrollo de la OIT desde su creación, luego de la incorporación de los Estados Unidos a la institución de Ginebra en 1934 las relaciones se hicieron más estrechas. Las delegaciones del continente a las conferencias internacionales demandaron un mayor reconocimiento de las realidades particulares de la región, las que no estaban contempladas en los principios constitutivos de la OIT, como el problema agrario o la situación de los indígenas. Estos problemas tuvieron la ocasión de plantearse en el trascurso de la Primera Conferencia Interamericana del Trabajo desarrollada en Santiago de Chile en 1936, donde las distintas delegaciones -entre ellas las sindicales- se manifestaron libremente respecto de los problemas laborales del continente, los mismos que, a decir de los mismos delegados, no podían ser resueltos en las instancias europeas (OIT, 1936; Herrera, 2013).

En materia de desarrollo del sindicalismo en América Latina, la historiografía se ha interesado en su evolución nacional y en los diferentes intentos -desde los años 1920- de unificación continental, marcados por el avance del comunismo y el desarrollo de la Guerra Fría luego de la Segunda Guerra Mundial (Poblete, 1946; Alba, 1964; Godio, 1985; Melgar, 1988). Sin embargo, se extrañan perspectivas analíticas que aborden el sindicalismo latinoamericano en el contexto de estudios regionales, comparados o transnacionales (Rodríguez, 2013). Si se considera el papel que han jugado las organizaciones internacionales en la formación del mundo contemporáneo (Iriye, 2002), existen muy pocos trabajos que destaquen la importancia que tuvo la OIT en la consolidación de sindicatos proclives a la Federación Sindical Internacional (FSI) durante los años 1920 (Yáñez, 2016), y los nexos que estableció a partir de fines de los años 1930 con la Confederación de Trabajadores de América Latina (CTAL) (Herrera, 2012).

Como una forma de reflexionar sobre los nexos entre el movimiento sindical latinoamericano y la OIT, nos 
interesa abordar de manera específica un capítulo un tanto desconocido de la acción de la institución de Ginebra en América Latina, como fue la misión de asistencia técnica que llevó a cabo en Venezuela en 1949. En noviembre de 1948 se produjo un golpe de Estado en ese país sudamericano, el cual derrocó al gobierno constitucional de Rómulo Gallegos, instalándose en su lugar una Asamblea Militar presidida por el general Carlos Delgado, la que limitó las garantías constitucionales y persiguió personalidades políticas y sindicales destacadas del país. La experiencia de Venezuela es importante porque permite estudiar la aplicación del convenio $\mathrm{n}^{\circ} 87$ sobre libertad sindical (1948), el cual es puesto a prueba por primera vez con este golpe de Estado. Además, nos permite acercarnos a las misiones de asistencia técnica implementadas por la OIT, que dan cuenta del contexto nacional e internacional en el cual se desarrollan y también de los equilibrios políticos que deben considerar para garantizar su buen resultado.

De esta forma, al encuentro de las dinámicas que impuso el sindicalismo, por una parte, y la presencia de la OIT en el continente, por otra, el presente artículo se estructura en una breve descripción de las primeras tentativas por unificar el movimiento sindical latinoamericano y los intereses de la OIT en ese proceso. Luego se concentra en la situación social de Venezuela durante los años 1930 y 1940, y cómo este país se transformó en un modelo del internacionalismo social impuesto desde Ginebra, para terminar con el estudio más detallado de la misión de asistencia sindical que se llevó a cabo en ese país en 1949.

\section{La OIT y la cuestión sindical}

Los primeros intentos por unificar el movimiento obrero latinoamericano pueden situarse en el año 1918 con la creación de la Confederación Obrera Panamericana (COPA), la cual contó con el apoyo de la Federación Americana del Trabajo (AFL). Sin embargo, y a pesar de su importancia simbólica, no pudo superar dos problemas concernientes a su fundación. En primer lugar, respondió a las tentativas de los Estados Unidos de controlar el movimiento obrero latinoamericano, apoyándose en las posiciones reformistas de algunos sectores obreros frente al avance del comunismo. En segundo lugar, al haber respondido a los intereses de la AFL, su influencia se vio reducida a América del Norte (Melgar, 1988, pp. 243-258).

De manera paralela, la FSI inició una política de acercamiento a las organizaciones obreras latinoamericanas ${ }^{2}$. El sindicalista español Francisco Largo Caballero elaboró un proyecto alternativo a la COPA promoviendo lazos entre el sindicalismo europeo y el latinoamericano. Según el historiador Ricardo Melgar, la proposición de Largo Caballero puede ser inscrita en los esfuerzos de los socialistas Pablo Iglesias y Jean Jaurès de abrir las puertas de la II Internacional a las vanguardias socialistas de América Latina, con el objetivo de disminuir el poder de la AFL frente a la FSI. En consecuencia, la UGT española propuso que se convocara en junio de 1920 en Madrid una conferencia obrera de los países de América del Sur, Portugal y España, con el fin de fundar una federación latinoamericana de trabajadores dependiente de la FSI.

Finalmente, la Internacional Sindical Roja (ISR) también buscó unificar el movimiento obrero del continente. A fines de 1927, los delegados sindicales de América Latina, invitados a la celebración del décimo aniversario de la Revolución Rusa, tuvieron en Moscú la Primera Conferencia Sindical Latinoamericana, que reunió a representantes de Argentina, Brasil, Colombia, Ecuador, México, Uruguay y Chile. Las resoluciones de la Confederación Sindical Latinoamericana (CSLA) se orientaron hacia la promoción de la unidad sindical del continente frente al imperialismo de los Estados Unidos, el apoyo al principio de la lucha de clases, el rechazo al sindicalismo reformista de la COPA y la formación de un amplio frente de trabajadores y de campesinos, entre otras resoluciones (Melgar, 1988, pp. 264-265). Desde la creación de la CSLA hasta su primer congreso en Montevideo en 1930 los sindicatos comunistas promovieron huelgas de carácter insurreccional y la formación de soviets locales y regionales. La línea política de la CSLA, coherente con los postulados de la ISR y de la III Internacional, se impuso como 
objetivo principal la revolución continental, y rechazó toda alianza con los sectores reformistas no solamente de la burguesía latinoamericana, sino también del movimiento obrero. Sin embargo, dos casos extremos marcaron tristemente el fracaso de la revolución continental. Primero, Agustín Farabundo Martí, dirigente del Partido Comunista, encabezó en El Salvador una revuelta en contra de la dictadura del general Maximiliano Hernández, que fue sometida a una represión sangrienta en 1932. En segundo lugar, la Unión Soviética apoyó en 1935 la rebelión de Luis Carlos Prestes y de la Alianza Nacional de Liberación del Brasil (ANL) en contra del gobierno de Getulio Vargas. La derrota de la rebelión condujo a la ANL a la ilegalidad, y esto fue acompañado por una ola de represión en contra de los comunistas, lo que reforzó al gobierno de Vargas hasta el golpe de Estado de 1945 (Godio, 1985, pp. 20-23).

Una razón que explica las condiciones favorables para la unidad sindical de la región es el cambio en la política de la Internacional Comunista a partir de su VII Congreso (1935); la misma pasó de una estrategia llamada clase contra clase a la formación de "frentes populares”, que consistió en una táctica de frente único antifascista, y que supuso la movilización de las masas y la alianza política con los sectores de la burguesía nacional. Luego de este cambio, la política de la CSLA favoreció la lucha en favor de la seguridad social, el aumento de salarios, la defensa de las conquistas ganadas por el movimiento obrero y la lucha en contra de la guerra imperialista. A pesar de esto, y en el nombre de la creación de frentes populares, la CSLA fue disuelta en 1936 de acuerdo a las resoluciones de la III Internacional. Estos esfuerzos de unificación del movimiento obrero continental se aceleraron a partir de septiembre de 1938, con la realización de un Congreso de los Sindicatos Obreros de América Latina que tuvo el apoyo de la Confederación de Trabajadores de México (CTM) y la participación de los principales representantes sindicales del continente. El congreso acordó constituir la Confederación de Trabajadores de América Latina (CTAL) ${ }^{\underline{3}}$ e instalar su sede central en México (Herrera, 2012). Como objetivos principales, la CTAL se planteó el progreso de la legislación laboral y la colaboración con otras organizaciones de carácter internacional, por lo que adoptó la siguiente resolución en lo concerniente a la OIT:

A los efectos de conseguir la ampliación y la aplicación de la legislación del trabajo en los países de América Latina la Confederación de Trabajadores Latinoamericanos colaborará activamente con la Oficina Internacional del Trabajo. Al mismo tiempo tratará por todos los medios a su alcance que a las Conferencias anuales concurran delegaciones completas y principalmente delegados de las entidades gremiales más representativas, genuinas y auténticas (OIT, 1939, p. 210).

El personaje más destacado de esta nueva organización continental fue el abogado y dirigente sindical mexicano Lombardo Toledano, quien la dirigió durante casi treinta años hasta su disolución en 1963. Toledano, marxista con una larga historia de lucha sindical, jugó un papel fundamental en el posicionamiento de la CTAL a nivel continental y en la profundización de sus relaciones con la OIT (Millon, 1966; Spenser, 2009). Esto le permitió ser el primer representante obrero de origen latinoamericano en formar parte del Consejo de Administración de la institución de Ginebra. Con ello, los esfuerzos en promover la unidad del movimiento obrero y la formulación de una política de alianza de clases iniciaron una nueva etapa en la consolidación de las relaciones entre el movimiento sindical continental y la OIT.

Si bien desde su creación la OIT se había destacado en la difusión de normas internacionales del trabajo, sorprende la reticencia para abordar la cuestión sindical, así como lo tardío en aprobar una convención sobre la materia (Bonvin, 1998), sobre todo si se considera la importancia de los sindicatos en la lucha por el mejoramiento de las condiciones laborales y la defensa de las conquistas ganadas. Frente a este vacío los delegados en las conferencias utilizaron el procedimiento de la impugnación como herramienta de lucha en contra de aquellas organizaciones que no eran representativas del movimiento obrero de sus países ${ }^{4}$.

Por su parte, el movimiento obrero latinoamericano tampoco había estado ajeno a la discusión sobre el papel 
de la OIT en la defensa de los derechos sindicales. Acorde con una política presente desde la Primera Conferencia Interamericana del Trabajo de Santiago de Chile en 1936, los representantes obreros demandaron un mayor acercamiento de la institución de Ginebra a los problemas propios de la región, así como una mayor democratización de sus instituciones y procedimientos (OIT, 1936). A partir de los años 1940 la unidad sindical del continente aparecía consolidada en torno a la CTAL, y sus relaciones con la OIT se hicieron evidentes en un punto esencial para el desarrollo de los países latinoamericanos: la función asignada a la industrialización como instrumento de transformación de las estructuras económicas y sociales. Tanto la OIT como la CTAL comprendieron que ni los progresos sociales ni el aumento de las fuerzas sindicales serían posibles en un continente con modalidades productivas tradicionales (coloniales), en el que, además, la mayoría de la población vivía en el campo y dependía de la agricultura de subsistencia. En principio, la industrialización permitiría transformar los métodos de producción de la agricultura, y ayudaría, con ello, al aumento de la producción de alimentos y a la reducción de la inflación (OIT, 1946, pp. 67-68). Además, la industrialización sería también funcional al movimiento sindical y a la acción de la OIT, porque ayudaría a consolidar la clase obrera y a mejorar las políticas sociales en el campo, al aumentar el número de trabajadores asalariados.

Si durante la Tercera Conferencia Interamericana del Trabajo, desarrollada en México en 1946, se ofreció un programa social y económico cercano a las posturas nacionalistas, antiimperialistas y favorables a la industrialización del continente, fue en gran parte porque sus posiciones se habían alimentado de los diagnósticos, de los estudios y de los informes elaborados por la OIT y, especialmente, por el desarrollo de las conferencias interamericanas del trabajo. El delegado peruano, por ejemplo, señaló en la Conferencia Interamericana del Trabajo de Santiago de Chile que

...para los elementos productores, la OIT representa el gran laboratorio de una nueva Humanidad, en la cual se trata de investigar y resolver los problemas del trabajo en forma científica, elevando el nivel de protección de los trabajadores, sin comprometer el desenvolvimiento de la economía mundial. Sus convenciones y recomendaciones vienen a significar, por eso, fórmulas de paz (OIT, 1936, pp. 93-94).

Por su parte, luego de algunos años de fructuosa relación, la OIT tenía solo palabras de reconocimiento a la labor realizada por la CTAL. La memoria de su director, Harold Butler, a la Tercera Conferencia Interamericana del Trabajo señaló lo siguiente:

Con la creciente influencia del movimiento sindical en estos países [América Latina], hay un evidente aumento del interés de trabajadores organizados, en las actividades de la Organización Internacional del Trabajo que ha encontrado su expresión, entre otras cosas, en un aumento de la presencia de representantes de los trabajadores de los países de América Latina en las Conferencias Internacionales del Trabajo convocadas desde la iniciación de la guerra (OIT, 1946, pp. 67-68).

De esta forma la OIT se mostró favorable a estos compromisos internacionales y dispuesta a continuar apoyando el desarrollo de un movimiento sindical autónomo y garante de los derechos de los trabajadores.

Una vez finalizada la Segunda Guerra Mundial se dieron las condiciones para discutir la aprobación de una convención sobre sindicalización. La unificación del movimiento obrero internacional en torno a la Federación Sindical Mundial (FSM) $)^{\frac{5}{}}$ en 1945 y la necesidad de la OIT de ganarse el apoyo de los obreros, ahora en un nuevo contexto internacional, dieron un nuevo impulso a la cuestión sindical. El hecho que aceleró la discusión sobre la materia fue la presentación de una memoria sobre los derechos de la organización obrera que hizo la FSM en 1947 al Consejo Económico y Social de las Naciones Unidas. En ella se solicitó la adopción de una resolución sobre la inviolabilidad del derecho sindical y la creación, en el seno de ese Consejo, de una comisión que resolviera sobre el tema, lo que cuestionaba a su vez el ámbito de 
atribuciones de la OIT. Sin embargo, el Consejo Económico y Social decidió reenviar la discusión al Consejo de Administración de la OIT, quien puso en tabla la cuestión de la libertad sindical en la conferencia del trabajo de 1947 (Bonvin, 1998, pp. 204-205).

En 1948 la conferencia internacional del trabajo aprobó la Convención n87 sobre libertad sindical, la que garantizaba "que los trabajadores y los empleadores, sin distinción y sin autorización previa, tienen el derecho de constituir las organizaciones que estimen convenientes, así como el de afiliarse a estas organizaciones, con la sola condición de observar los estatutos de las mismas”. Además, aseguraba a las organizaciones de trabajadores y de empleadores la elaboración de sus estatutos y de sus reglamentos administrativos, la elección libre de sus representantes, la organización de su gestión y la formulación de su programa de acción. Las organizaciones tenían también el derecho de constituir federaciones y confederaciones, y de afiliarse a ellas. Esta convención fue completada con la convención n 98 de 1949, sobre el derecho de organización y de negociación colectiva.

Las discusiones normativas y valóricas que se tuvieron a la hora de aprobar esta convención apuntaron igual que en la década de 1920 - a resolver si el derecho de sindicalización debía ser obligatorio para todos los trabajadores y si el reconocimiento legal de los sindicatos debía o no estar supeditado al respeto de las leyes nacionales, teniendo en cuenta que éstas podían ir en contra de las disposiciones de una convención sobre la materia. Los acuerdos apuntaron, por un lado, a conciliar la libertad y el compromiso de los trabajadores en la lucha por sus derechos laborales y, por otro, a garantizar que las legislaciones nacionales no atentaran en contra de las organizaciones sindicales. Sin embargo, esta posición de compromiso no había resuelto un problema mayor: ¿cómo se debía actuar frente a un gobierno de facto que hubiese iniciado un proceso de persecución del movimiento sindical y que desde un punto de vista de procedimiento no había ratificado la convención $n^{\circ}$ 87? Lo importante del caso de Venezuela es que puso a prueba esta convención, lo que nos permite analizar el grado de compromiso de la OIT en la defensa del movimiento obrero, en una nueva época de internacionalismo, marcada por la Declaración Universal de los Derechos Humanos (1948).

\section{La situación de Venezuela en los años 1930 y 1940}

A partir de la segunda mitad de los años 1920, Venezuela comenzó a participar más activamente en las organizaciones satélite de la Sociedad de las Naciones, particularmente en las conferencias internacionales del trabajo, lo que se puede explicar como parte de una política de apertura del gobierno de Juan Vicente Gómez (1922-1929). En este contexto, el país sudamericano se comprometió en un programa de política social y aprobó la primera Ley del Trabajo en 1928, la cual agrupó las disposiciones existentes en los códigos de minas, de comercio y civil. Sin embargo, esta ley tuvo problemas en su implementación, especialmente por la falta de reglamentación. Además, algunas de sus disposiciones eran contrarias a las normas internacionales, como la jornada de trabajo de nueve horas o la prohibición de la representación internacional de las asociaciones profesionales (Caldera, 1939, p. 95).

En los años 1930, Venezuela presentaba un fuerte crecimiento económico gracias a la producción de petróleo, con el consiguiente desarrollo de un movimiento obrero que provocaba conflictos serios al momento de exigir condiciones de trabajo según los estándares internacionales. El gobierno del General Eléazar López (1935-1941) solicitó a la OIT su apoyo en la preparación de un nuevo código laboral. Ésta nombró a David Blelloch -jefe adjunto de la sección de Derecho y de Inspección del Trabajo de la OIT- para llevar a cabo la misión de asistencia técnica ${ }^{6}$. Blelloch tenía una amplia experiencia en la materia y había participado en la Primera Conferencia Interamericana del Trabajo de Santiago de Chile, donde había establecido vínculos con autoridades y expertos locales ${ }^{\underline{7}}$. 
La falta de claridad sobre el dominio de acción del funcionario de la OIT, así como la amplitud de temas que debían ser abordados, hizo difícil la redacción de un proyecto en armonía con las elites nacionales. En este contexto Blelloch y la comisión encargada de la redacción del nuevo proyecto recurrieron a la actualización de ciertas disposiciones contenidas en el Código del Trabajo de 1928 y en las mismas convenciones internacionales (Caldera, 1936, p. 98). ¿Qué disposiciones presentes en las convenciones formaron parte del proyecto de 1936? En la época, entre 1932 y 1933, Venezuela había ratificado cuatro convenciones, especialmente sobre aspectos secundarios de la reglamentación laboral: la convención n 4 (1919) sobre el trabajo nocturno de mujeres; la convención $n^{\circ} 6$ (1919) sobre el trabajo de menores; la convención $n^{\circ} 13$ (1921) sobre el uso de la cerusa en la pintura; y la convención $n^{\circ} 27$ (1929) sobre la indicación del peso de los fardos transportados en barco.

Uno de los miembros de la comisión que redactó la Ley del Trabajo de 1936 señaló que ésta contenía preceptos de las convenciones internacionales, del código laboral chileno y mexicano, y de la Ley del Trabajo venezolana de 1928. La ley de 1936 introdujo también algunas modificaciones compatibles con los principios contenidos en las convenciones de Ginebra, especialmente en materia de jornada de trabajo (ocho horas) y de sindicalización obrera. Sin embargo, la legislación de 1936 fue objeto de numerosas críticas. La más importante fue aquella que consideró que las disposiciones sobre seguridad social debían estar contenidas en un proyecto de reglamentación autónomo, para lo cual se envió al Congreso un proyecto de ley sobre la materia en 1938. Este proyecto fue redactado casi por completo por Antonio Zelenka, funcionario de la OIT. Finalmente, una comisión designada por el ministro del Trabajo, y con la asistencia técnica de Wilfred Jenks, miembro del servicio jurídico de la OIT, presentó un nuevo proyecto de código laboral, muy ambicioso, que contenía 951 artículos (Caldera, 1936, p. 115).

La participación de estas delegaciones de la OIT en Venezuela, muestra su importancia como nación modelo en la asistencia internacional, sin desconocer que era el segundo país productor de petróleo luego de los Estados Unidos ${ }^{8}$. Con un número total de 4.700 .000 habitantes, su población activa era de 1.240 .000 en 1941, es decir, el 32,2\% del total. La mitad de esa población activa trabajaba en la agricultura y 16,7\% solamente lo hacía en la industria. La producción de petróleo había permitido un desarrollo industrial progresivo, especialmente en el área de la refinería y de los productos derivados. De este modo, una parte creciente del presupuesto dependía de los ingresos provenientes directa o indirectamente del petróleo. La política gubernamental consistió en reinvertir parte de esos ingresos en el desarrollo de otros sectores de la economía nacional, como la agricultura y las obras públicas. Dos instituciones fueron creadas para tal efecto: el Banco Agrario y la Corporación de Promoción. Las condiciones de los trabajadores de la industria petrolera eran las mejores del país y con los salarios más elevados. Por ejemplo, si los obreros agrícolas ganaban 4 bolívares por día y los trabajadores ocupados en las obras públicas entre 5 y 7, el salario base de los trabajadores del petróleo variaba entre 8 y 12 bolívares. Aunque estos últimos representaban solamente el 4,8 \% de la población activa, las ventajas obtenidas en las convenciones colectivas servían de referencia a las otras áreas de la producción (Bergquist, 1988).

Según el informe elaborado por la misión de asistencia técnica de la OIT, estas condiciones -un pobre desarrollo industrial, estrechamente asociado al petróleo y sin efectos en la población económicamente activa- explicaban el lento desarrollo del movimiento sindical en Venezuela, pese al aumento que había mostrado a partir de la aprobación del Código del Trabajo de 1936 (Vitale, 1981). Éste había establecido la jornada de ocho horas, el derecho a sindicalización y a huelga, junto con los mecanismos de conciliación y arbitraje. Una demostración concreta de las consecuencias de la legislación de 1936 fue la huelga petrolera - la más importante de esos años-, que duró cuarenta y dos días. Aunque no tuvo efectos inmediatos en el mejoramiento de las condiciones laborales, la huelga manifestó las potencialidades de la organización sindical y mostró a las compañías petroleras que sin el reconocimiento de las demandas de los trabajadores 
los conflictos aumentarían (Bergquist, 1988). A esta ola de sindicalización ayudó la Constitución de 1947, que permitió el derecho de asociación, aunque con dos indicaciones: primero, que ese derecho se dirigiera a “fines lícitos”, es decir respetando las leyes de orden público, y segundo, que las leyes y reglamentos no limitaran los derechos garantizados por la Constitución (OIT, 1950, p. 53). La constitución garantizó, en efecto, una serie de derechos que comprendían tres aspectos fundamentales: el fuero sindical, el derecho a negociar convenios colectivos y, por último, el derecho a huelga, salvo en los servicios públicos que la ley acordara.

La represión al movimiento político y sindical durante el gobierno del general Eléazar López (1935-1941) limitó la acción de la Confederación Venezolana de Trabajadores (CVT), creada en 1936 y disuelta en 1944, acusada de estar infiltrada por los comunistas (Urquijo, 2004). La unificación del movimiento obrero venezolano se produjo en 1945 con el apoyo de la CTAL, creándose la Federación de Trabajadores de Venezuela (FTV), que intentó integrar las dos grandes corrientes políticas: El Partido Comunista y El Partido de Acción Democrática ${ }^{9}$. Sin embargo, las dos corrientes continuaron dividiendo al movimiento obrero durante la presidencia de Rómulo Betancourt (1945-1948)ํㅜㄹ y de Rómulo Gallegos (1948).

Durante el gobierno de Betancourt, las autoridades mostraron gran reticencia en reconocer los sindicatos independientes, lo que condicionó la búsqueda de un empleo -especialmente en las obras públicas y en las empresas del Estado- a la inscripción en un sindicato favorable al gobierno o al Partido de Acción Democrática. Este proceso se vio reforzado con la creación de la Confederación de Trabajadores de Venezuela (CTV) en 1947, cuyos principales dirigentes eran adherentes al Partido de Acción Democrática (Urquijo, 2004). Esta relación estrecha entre la corriente sindical y la política debía crear dificultades importantes al momento de diferenciar el marco de acción de cada una de ellas, en especial si un número no menor de dirigentes sindicales eran al mismo tiempo miembros de la Cámara de Diputados y del Senado. Si bien la delegación de la OIT no cuestionó mayormente esta relación estrecha entre lo sindical y lo político, ni tampoco las autoridades militares invocaron el argumento de ilegalidad de los sindicatos que tenían estos vínculos, la experiencia demostraba que estos lazos sí existían, eran estrechos y podían dar origen a una persecución de carácter político con consecuencias inesperadas en el ámbito sindical. Estos lazos son consignados en el informe que elaboró la delegación de la OIT a propósito de su visita a los sindicatos venezolanos:

\section{ORGANIZACIONES SINDICALES Y CONTROL DE LOS PARTIDOS POLÍTICOS EN 1949}

\begin{tabular}{|l|l|}
\hline La Confederación del Trabajo de Venezuela: & Partido de Acción Democrática \\
\hline Los sindicatos comunistas (rojos): & Partido Comunista \\
\hline Los sindicatos comunistas (negros): & Partido Comunista Independiente \\
\hline Los sindicatos social-cristianos: & Partido COPEI \\
\hline
\end{tabular}

Fuente: OIT (1950).

La delegación era consciente del peligro de entablar relaciones estrechas entre la acción política y la sindical, en un país donde los cambios de gobierno eran frecuentes y a menudo inesperados, incluso si se ratificaba la legalidad de los sindicatos tal y como existían al momento de la toma del poder por parte de la Junta Militar.

En suma, la delegación diagnosticó que la desorganización del movimiento sindical era evidente y que la situación se había hecho más grave a partir de fines del mes de febrero de 1949 con la disolución de la CTV, acusada de tener actividades políticas no autorizadas. 


\section{La misión de asistencia sindical en Venezuela, 1949}

El origen de la misión de asistencia técnica en Venezuela se debe al interés de León Johaux -representante sindical obrero del Consejo de Administración de la OIT- de poner en la tabla de discusión la situación sindical de ese país luego del golpe de Estado de noviembre de 1948. La situación venezolana fue también abordada en la Cuarta Conferencia Interamericana del Trabajo, desarrollada en 1949.

En este ambiente de presión internacional, la Asamblea Militar invitó a la OIT a visitar el país para evaluar la situación en el dominio de sus atribuciones, especialmente en tres aspectos: las condiciones de trabajo en los diferentes ámbitos de la producción; la legislación protectora de los trabajadores, y, finalmente, el desarrollo y funcionamiento de las organizaciones sindicales. El director de la OIT, David Morse, aceptó la invitación del gobierno venezolano, pero a condición de "poner a disposición [de la delegación] todas las facilidades necesarias y, especialmente, darle la facultad de discutir libremente con los representantes debidamente acreditados de las organizaciones de empleadores y de trabajadores” (OIT, 1950, p. 2). Jef Rens, subdirector general de la OIT, fue nombrado jefe de la delegación, lo que refleja la importancia dada a la visita $\frac{11}{}$, la cual tuvo lugar entre el 22 de julio al 1 de septiembre. Jef Rens no se limitó, como estaba previsto, a instalar la delegación y a organizar sus primeras actividades, sino que además asumió una parte activa durante todo el estudio. La comisión de recepción estableció un programa de visitas que permitió a la delegación recorrer una buena parte del país, entrevistar a numerosas personalidades y conocer las reparticiones oficiales. Los funcionarios de la OIT visitaron, además de Caracas -capital nacional-, trece estados de los veinte que constituían el país en aquella época. Para completar el panorama, la misión pudo reunir una abundante documentación, aunque ciertas estadísticas oficiales eran inexistentes y otras estaban en plena organización. La lista de reuniones que acordó la delegación es amplia y comprende entrevistas con funcionarios de gobierno -incluida la Junta militar que gobernaba el país-, dirigentes sindicales y empresariales, embajadores de gobiernos europeos, visitas a diferentes fábricas, inspectores del trabajo, entre otros $\frac{12}{}$.

El estudio de las condiciones de trabajo y de vida de la población obrera constituía otra de las tareas confiadas a la delegación de la OIT (capítulo III del Informe). Para la institución de Ginebra, Venezuela podía mostrar un historial importante de disposiciones de protección a los trabajadores, especialmente a partir de la aprobación del Código del Trabajo de 1936. En relación a la seguridad social, la protección se dividía en tres categorías principales: a) Los seguros sociales obligatorios, instaurados en 1940, que incluían dos ramas; el seguro de enfermedad y maternidad, y el seguro de accidentes del trabajo y de enfermedades profesionales (los trabajadores agrícolas, a domicilio y domésticos, no estaban cubiertos por el seguro); b) las obligaciones impuestas a los empleadores en materia de seguridad social -de acuerdo a las disposiciones de la Ley del Trabajo o de las convenciones colectivas- las cuales tratan especialmente de las condiciones de higiene y de seguridad en la industria, algunos cuidados médicos a los trabajadores y a los miembros de su familia, la reparación de riesgos profesionales, y las indemnizaciones por despido y antigüedad (los trabajadores agrícolas estuvieron cubiertos a partir de 1945); c) Los servicios de la salud pública, en particular los servicios hospitalarios del Ministerio de Salud y de la Previsión Social (OIT, 1950, pp. 134135).

La delegación propuso extender progresivamente los beneficios de la seguridad social al conjunto de la población y uniformar su aplicación, además de consolidar sus ventajas en áreas prioritarias como el seguro de cesantía y de jubilación. Además, era necesario mejorar el funcionamiento de la Inspección del Trabajo, en especial en la formación de su personal y de los medios para el cumplimiento de sus funciones. Frente a la envergadura de las tareas planteadas, la delegación propuso una política de conciliación entre los diferentes sectores sociales, en la que la libertad sindical sería condición previa de dicha colaboración. En el aspecto sindical, el informe de la OIT sugería resguardar los sindicatos de las vicisitudes de la vida política y reforzar su prestigio y eficacia estableciendo una demarcación más clara entre sus actividades puramente sindicales y 
las políticas, y evitando la represión y su dependencia del Estado (OIT, 1950, p. 178). A la luz de los hechos mostrados por el propio informe, la delegación entregó algunas propuestas con el objeto de permitir el funcionamiento normal de los sindicatos:

1. Eliminación de la prohibición de la reelección de los antiguos miembros de los comités directores de los sindicatos.

2. Supresión de la autorización previa para la realización de asambleas sindicales.

3. Restablecimiento del derecho de los sindicatos a agruparse libremente en federaciones y confederaciones nacionales.

4. Restablecimiento del ejercicio libre del derecho a huelga y al lock-out en los límites fijados por la Ley del Trabajo.

5. Apoyo en la colaboración de todos los factores de la producción representados por organizaciones sólidas e independientes de empleadores y de trabajadores.

Al conocerse el informe redactado por la delegación se abrió un debate inesperado entre el gobierno venezolano y la OIT, lo que puso a prueba la naturaleza del procedimiento de investigación llevado a cabo y, por lo tanto, la legitimidad de sus conclusiones. El primer aspecto conflictivo se centraba en la relación con el objetivo de la misión de asistencia y con su mandato. Si para el gobierno venezolano la delegación comprendía "un estudio destinado a proveer a la OIT de informaciones completas e imparciales y no de una investigación cuyo informe oficial sería publicado” (Gobierno de Venezuela, 1951, p. 3), la institución de Ginebra había ido más allá del ámbito de su competencia, y había transformado la visita en una comisión de investigación que debía ser establecida, en todo caso, según determinados principios reglamentarios. Para el gobierno venezolano las comisiones de investigación estaban previstas en casos muy determinados, por ejemplo, para el caso en que un Estado -también una organización profesional de empleadores o de trabajadores- acusaba a otro Estado de haber infringido las obligaciones emanadas de una convención que hubiera ratificado. De acuerdo al gobierno venezolano, ninguna acusación había sido presentada por la violación de la convención sobre sindicalización y menos una acusación de ese género se había comunicado a las autoridades venezolanas.

El segundo aspecto conflictivo se produjo a propósito de la publicación del informe, lo que no había sido considerado en un primer momento. Nueve meses después de la visita, en mayo de 1950, la OIT entregó al gobierno venezolano un documento de 194 páginas donde exponía los resultados de su misión. Las autoridades venezolanas no tuvieron tiempo de hacer llegar sus objeciones al informe, aunque la OIT ofreció todas las garantías de difusión de dichas observaciones. Entre las críticas estaba el hecho de que en ningún momento el gobierno había sido consultado con el fin de saber si deseaba o no que el informe fuese publicado. Además, se criticaron las apreciaciones sociológicas, étnicas e históricas presentes en él, junto con los errores de juicio sobre diferentes temas. De manera simbólica, las autoridades cuestionaron el uso un tanto tendencioso de un panel de fotografías presentes en el informe, prueba del poder de la imagen como elemento de denuncia. Por último, el gobierno venezolano cuestionó que se relegaran a un segundo plano los avances en la legislación social y en la protección acordada a los trabajadores por causa de la atención prioritaria dada a la situación sindical (Gobierno de Venezuela, 1951, pp. 5-6).

El gobierno venezolano no pudo evitar la publicación del informe, fijada para el 30 de junio de 1950. La OIT comunicó a las autoridades que publicaría sus observaciones como complemento del informe, siempre y cuando llegaran en el tiempo previsto, o como documento distinto, si llegaban posteriormente a la fecha acordada. 
Lo que muestra la experiencia de Venezuela es la dificultad en avanzar en el cumplimiento de la legislación social en aquellos países refractarios a la intervención de la OIT, o que presentaban condiciones políticas complejas, propias de una dictadura militar. Algunos gobiernos utilizaron estas misiones para dirigir una ola de nacionalismo frente a la intervención de un organismo extranjero, lo que marca un retroceso en el cumplimiento de los compromisos internacionales en materia laboral. Las autoridades venezolanas reiteraron en sus observaciones al informe de la delegación de la OIT lo siguiente:

Resulta que la soberanía exterior de un Estado se apoya sobre su autonomía y que toda entidad extranjera a ese Estado que critica o censura su organización interior en el dominio político, atenta, en una u otra medida, contra los derechos soberanos de ese Estado. Es por esta razón que en los instrumentos internacionales fundamentales, como la Carta de las Naciones Unidas, se encuentra consagrado el principio de la no intervención en los asuntos internos de los Estados Miembros, salvo en caso de amenaza para la paz y la seguridad internacional; la misión no parece haber tenido en cuenta ese principio que es la verdadera base de la colaboración internacional (Gobierno de Venezuela, 1951, p. 10).

$\mathrm{Al}$ parecer, con la experiencia venezolana, la OIT terminó por comprender las dificultades para incorporar a un país en el camino de la cooperación sin tener en cuenta las opiniones de las propias autoridades nacionales.

Frente al impase provocado por el rechazo del gobierno venezolano a aceptar las conclusiones del informe de la OIT y, lo más grave, su cuestionamiento al procedimiento de investigación, el Consejo de Administración de la institución de Ginebra debió revisar sus modalidades de aplicación de la convención sobre libertad sindical, en especial frente a aquellos países que no habían ratificado la convención o que se mostraban enemigos de toda injerencia de una institución extranjera. En 1951, se formó el Comité de Libertad Sindical para examinar las acusaciones en contra de un Estado que no cumpliera con los principios contenidos de la convención sobre libertad sindical o negociación colectiva, aunque hubiera o no ratificado estas convenciones. Las quejas formuladas por las organizaciones de trabajadores o de empleadores contra un Estado miembro serían examinadas por el Comité de Libertad Sindical, el cual estaría compuesto por un presidente y tres representantes de los gobiernos, empleadores y trabajadores (Gavel, Duplesseis y Gernigon, 2001).

\section{Conclusiones}

Las misiones de asistencia técnica en materia de trabajo muestran las complejidades y dificultades en manejar variables que no habían sido consideradas hasta ese momento, como la situación política, la oposición de las elites locales, el sentimiento nacionalista, entre otros aspectos. Las misiones de asistencia aparecen también como espacios propicios para reforzar la experticia de la OIT. En este contexto, la investigación llevada a cabo en Venezuela sobre la situación sindical es importante y reveladora por dos razones. En primer lugar, desde el punto de vista del procedimiento, la discusión normativa en torno a la convención sobre libertad sindical tendió a conciliar la libertad de los trabajadores en la lucha por sus derechos laborales y garantizar que la legislación nacional no limitara el desarrollo de las organizaciones sindicales. No obstante, esta posición de compromiso no había resuelto un problema mayor: ¿cómo actuar frente a un gobierno que inicia un proceso de persecución del movimiento sindical y que desde un punto de vista de procedimiento no ha ratificado la convención $n^{\circ}$ 87? La OIT debió reorganizar sus protocolos de elaboración de normas y adaptarlos a las condiciones complejas que América Latina ofrecía. En segundo lugar, el caso venezolano muestra una nueva forma de abordar los problemas que emergen a nivel nacional. En efecto, el movimiento de contestación iniciado por la clase política y sindical venezolana en el interior del 
país y en el exilio hizo aparecer la solidaridad internacional, lo que se explica, precisamente, por los lazos construidos entre los sectores políticos y sindicales venezolanos con las instituciones internacionales durante los años 1930 y 1940, especialmente con la Unión Panamericana, la OIT, la CTAL y la FSM.

El conjunto de reflexiones que hemos ofrecido en este artículo muestra la riqueza de experiencias que el continente ofreció al internacionalismo de la época y el carácter de laboratorio social que tuvo para la OIT.

\section{Notas}

1 En los últimos años se ha producido una renovación en los estudios de la OIT a partir de los enfoques transnacionales y comparados. Véase Van Daele (2010); Lespinet-Moret y Viet (2011); Aglan, Feiertag y Kévonian (2011); Kott y Droux (2012); Herrera y Wehrli (2011). Sobre los primeros nexos entre la OIT y América Latina véase Herrera y Herrera (2013).

2 La FSI se creó en 1901 como la expresión sindical de la II Internacional, adscribiéndose ideológicamente a las corrientes reformistas y socialdemócratas europeas. También llamada Internacional de Ámsterdam por la sede de su Secretariado General, funcionó hasta 1945, cuando resolvió integrarse a la Federación Sindical Mundial (FSM).

$\underline{3}$ La CTAL tuvo una orientación ideológica de tipo internacional sindicalista, aunque de colaboración con las corrientes progresistas del periodo, y buscó establecer alianzas con un amplio espectro de partidos, tanto comunistas como socialistas, radicales y liberales, con el fin de fortalecer la democracia.

4 Durante los años 1920 habían sido históricas las impugnaciones a las delegaciones obreras de Italia y de Rusia. En el caso latinoamericano, las delegaciones obreras de la Argentina fueron impugnadas en distintos momentos (Yáñez, 2014).

$\underline{5}$ La FSM se define ideológicamente como democrática, antiimperialista y al servicio de la unidad obrera mundial, buscando la consolidación de la paz luego de la Segunda Guerra Mundial. Inicio una lucha por la independencia de las colonias de Asia y África. La CTAL fue un aliado relevante de la FSM, al ser elegido Vicente Lombardo Toledano como vicepresidente en su congreso constituyente de octubre de 1945 en París.

6 "Invitation from Venezuelan Government to send ILO expert in labor legislation, (Blelloch’s mission, 1936)”, Cabinet Harold Butler, XT 63/1/1, Ginebra, marzo 1936.

Z Archivo de la OIT, P760, Expediente David Blelloch.

$\underline{8}$ El único estudio que conocemos que ha destacado la importancia de Venezuela en el modelo de asistencia técnica de la OIT es aquel de Plata (2013, pp. 127-160).

9 El Partido Comunista de Venezuela fue fundado en 1931 por Juan Bautista Fuenmayor, entre otros. El Partido de Acción Democrática fue fundado en 1941 por Rómulo Gallegos y Rómulo Betancourt, siendo un partido de tendencia socialdemócrata.

10 Rómulo Betancourt apoyó el golpe de Estado de 1945 que derrocó al gobierno de Isaías Medina, y fue elegido presidente provisorio de Venezuela.

11 Para la organización de la visita y los primeros contactos, así como los documentos, véase Archivo de la OIT, MI 321, Missions to Venezuela, 06-1949.

12 "Liste des visites et entrevues de la mission”, Archivo de la OIT, MI 321, Missions to Venezuela, 061949. 


\section{Bibliografía}

Aglan, A., Feiertag, O y Kévonian, D. (dir.) (2011). Humaniser le travail. Régimes économiques, régimes politiques et Organisation internationale du travail (1929-1969). Bruxelles: Peter Lang.

Alba, V. (1964). Historia del Movimiento Obrero en América Latina. México: Libros Mexicanos.

Bergquist, C. (1988). Los trabajadores en la historia latinoamericana. Estudios comparativos de Chile, Argentina, Venezuela y Colombia. Bogotá: Siglo XXI.

Bonvin, J. M. (1998). L’Organisation internationale du travail. París: PUF.

Caldera, R. (1939). Derecho del Trabajo. Caracas: Tipografía La Nación.

Daele, J. V. et al., (dir.) (2010). ILO Histories: Essays on the International Labour Organization and Its Impact on the World during the Twentieth Century. Berna: Peter Lang.

Ferreras, N. (2011). Entre a expansão e a sobrevivência: a viagem de Albert Thomas ao Cone Sul da América. Antíteses, 4(7), 127-150.

Gavel, E, Duplesseis, I. y Gernigon, B. (2001). Le Comité de la liberté syndicale: quel impact depuis sa création?. Ginebra: OIT.

Gobierno de Venezuela (1951). La Liberté syndicale et les conditions de travail au Venezuela. Observations du Gouvernement du Venezuela sur le rapport de la mission du Bureau international du Travail. Ginebra: OIT.

Godio, J. (1985). Historia del movimiento obrero latinoamericano. Caracas: Editorial Nueva Sociedad.

Herrera, P. (2012). Vicente Lombardo Toledano: nexo entre los obreros latinoamericanos y la OIT. Trabajadores, 91, 23-28.

Herrera, P. (2013). La primera conferencia regional del trabajo en América: su influencia en el movimiento obrero, 1936. En F. Herrera y P. Herrera (coord.), América Latina y la OIT. Redes, cooperación técnica e institucionalidad social (1919-1950) [179-219]. Morelia: Universidad Michoacana San Nicolás de Hidalgo.

Herrera, F. y Wehrli, Y. (2011). Le Bureau international du travail et l'Amérique latine durant l'entre-deuxguerres. En L. Lespinet-Moret y V. Viet (dir.), L'Organisation internationale du travail. Origine, développement, avenir [157-166]. Rennes: PUR.

Herrera, F. y Herrera, P. (coord.) (2013). América Latina y la OIT. Redes, cooperación técnica e institucionalidad social (1919-1950). Morelia: Universidad Michoacana San Nicolás de Hidalgo.

Iriye, A. (2002). Global Community. The role of international organizations in the making of the contemporary world. Berkeley: California University Press.

Kott, S. y Droux, J. (dir.) (2012). Globalizing Social Rights: The International Labor Organization and beyond. New York: Palgrave.

Lespinet-Moret, I. y Viet, V. (dir.) (2011). L’Organisation internationale du travail. Origine, développement, avenir, Rennes: PUR.

Melgar, R. (1988). El movimiento obrero latinoamericano. Historia de una clase subalterna. Madrid: Alianza Editorial.

Millon, R. (1966). Mexican marxist. Vicente Lombardo Toledano. Chapel Hill: University of North Carolina Press. 
OIT (1936). Conferencia del Trabajo de los Estados de América, Acta de las Sesiones, Ginebra: OIT.

OIT (1939). Informe acerca de las medidas tomadas para dar cumplimiento a las resoluciones adoptadas por la Conferencia de Santiago de Chile. Ginebra: OIT.

OIT (1946). Tercera Conferencia del Trabajo, Memoria del Director. Montreal: OIT.

OIT (1954). L'assistance technique. Ginebra: OIT.

Plata V. (2013). La difusión de las normas internacionales del trabajo en Venezuela, 1936-1939: una práctica de cooperación técnica internacional en la OIT. En F. Herrera y P. Herrera (coord.), América latina y la OIT. Redes, cooperación técnica e institucionalidad social (1919-1950) [113-144] Morelia: Universidad Michoacana San Nicolás de Hidalgo.

Poblete, M. (1946). El movimiento obrero latinoamericano. México: FCE.

Rodríguez, M. (2013), Constructing Labour Regionalism in Europe and the Americas, 1920s-1970s, IRSH, 58, 39-70.

Spenser, D. (2009), Vicente Lombardo Toledano envuelto en antagonismos internacionales. Revista Izquierdas, 4. Recuperado de http://izquierdas.cl/revista/wp-content/uploads/2011/07/spenser.pdf (recuperado el 12 de marzo de 2015).

Urquijo, J. (2004). El movimiento obrero de Venezuela. Caracas: OIT, UCAB, INAESIN.

Vitale, L. (1981). Notas para una historia del movimiento obrero venezolano. UCV. Recuperado de http ://www.archivochile.com/Ideas Autores/vitalel/3lvc/03lvcmovsoc0012.pdf (recuperado el 14 de abril de 2015).

Yáñez, J. C. (2000). Chile y la Organización Internacional del Trabajo (1919-1925). Hacia una legislación social universal. Revista de Estudios Histórico-Jurídicos, 22, 317-332.

Yáñez, J. C. (2014). L’OIT y l'Amérique du Sud. La construction d'un laboratoire social régional (19191949). París: Tesis para optar al grado de Doctor en Historia, EHESS-París

Yáñez, J. C. (2016). La OIT en América del Sur. El comunismo y los trabajadores chilenos (1922-1932). Santiago de Chile: Ediciones Universidad Alberto Hurtado. 